\section{Cotitis} University of Calgary, Calgary, Alberta, Canada

${ }^{2}$ Department of Pathology and Laboratory Medicine, University of Calgary, Calgary, Alberta, Canada

${ }^{3}$ Department of Radiology, University of Calgary, Calgary, Alberta, Canada

${ }^{4}$ Department of Surgery, University of Calgary, Calgary, Alberta, Canada

${ }^{5}$ Department of Community Health Sciences, University of Calgary, Calgary, Alberta, Canada

\section{Correspondence to}

Dr Kerri A Johannson, Department of Medicine, University of Calgary, Calgary, AB T2N 1N4, Canada; kerri.johannson@ahs.ca

Received 13 February 2018 Revised 16 May 2018

Accepted 21 May 2018 Published Online First 8 June 2018

\title{
Contralateral recurrence of inflammatory myofibroblastic tumour of the lung 10 years after pneumonectomy
}

\author{
Daniel C Vis, ${ }^{1}$ Margaret M Kelly, ${ }^{2}$ Andrew G Lee, ${ }^{3}$ Gary Gelfand, ${ }^{4}$ Kerri A Johannson ${ }^{1,5}$
}

\section{INTRODUCTION}

Inflammatory myofibroblastic tumour (IMT) is a rare form of inflammatory pseudotumour of the lung. Complete surgical resection is the treatment of choice, with corticosteroid-based medical therapy reserved for cases where complete resection is not possible. ${ }^{1}$ To date, the majority of recurrent cases have occurred subsequent to incomplete resection or after discontinuation of steroids when used as primary therapy. ${ }^{12}$ There is a paucity of data guiding the follow-up of patients who undergo complete resection. In addition, the efficacy of oral steroids in the treatment of IMT remains poorly characterised and the optimal duration of therapy is unknown.

\section{CASE PRESENTATION}

A 46-year-old lifetime non-smoker presented in December 2015 with dyspnoea, fatigue, night sweats and weight loss. A chest X-ray demonstrated a new left lower lobe mass. His history was significant only for a right pneumonectomy in June 2005 for a large inflammatory pseudotumour. This pneumonectomy was performed for definitive surgical management, as the mass was adjacent to the pulmonary artery, with concern for pulmonary artery invasion, and for definitive diagnosis. Given that a definitive surgical option was available in this otherwise healthy man, and that invasion of the pulmonary artery could lead to catastrophic complications, oral corticosteroid therapy was not pursued prior to the pneumonectomy. Postpneumonectomy, he was assessed annually with a chest X-ray and for the subsequent 10 years, only postsurgical changes were noted. No medical therapy was pursued postpneumonectomy due to the low anticipated risk of recurrence after complete surgical resection, and lack of evidence supporting a preventative pharmacological approach.

CT scan of the chest showed a dense mass in the superior segment of the left lower lobe (figure 1A). Percutaneous biopsy of the mass revealed a fibroinflammatory lesion consistent with IMT, with characteristic findings of spindled cells in a fascicular arrangement and abundant lymphoplasmacytic infiltration (figure 2). Notably, the criteria for IgG4-related disease were not met, and ALK-1 was negative by immunohistochemistry and fluorescence in situ hybridisation testing. Given that the mass could not be resected due to prior pneumonectomy, oral prednisone was started at a dose of $0.5 \mathrm{mg} / \mathrm{kg} /$ day. Dramatic improvement occurred symptomatically and radiographically, with steady improvement of the focal mass-like lesion in the superior segment of the left lower lobe at 5 months (figure 1B).

\section{DISCUSSION}

To our knowledge, this is the first report of contralateral recurrence of IMT after pneumonectomy and the longest time period to recurrence after complete surgical resection. Recurrent contralateral disease has been reported in patients undergoing complete resection without pneumonectomy, ${ }^{3}$ but we believe this case presents a unique entity, as recurrent
Check for updates

To cite: Vis DC, Kelly MM, Lee $A G$, et al. Thorax 2018:73:1089-1090.

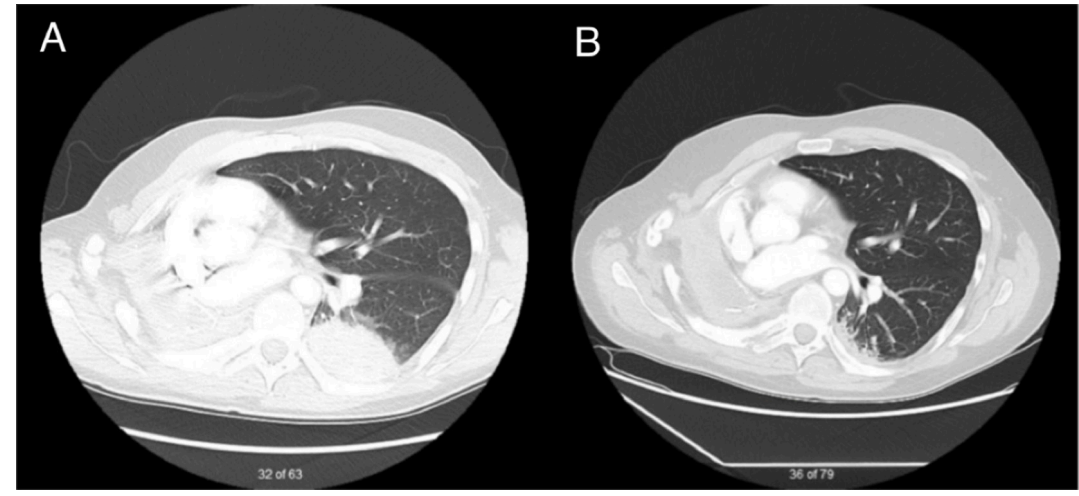

Figure 1 Contrast-enhanced CT of the chest demonstrating (A) left lower lobe mass-like lesion, $6.8 \mathrm{~cm} \times 4.0 \mathrm{~cm}$ in anteroposterior dimension in a postpneumonectomy thorax, and (B) marked reduction in size of the left-sided lesion after 5 months of oral corticosteroids. 

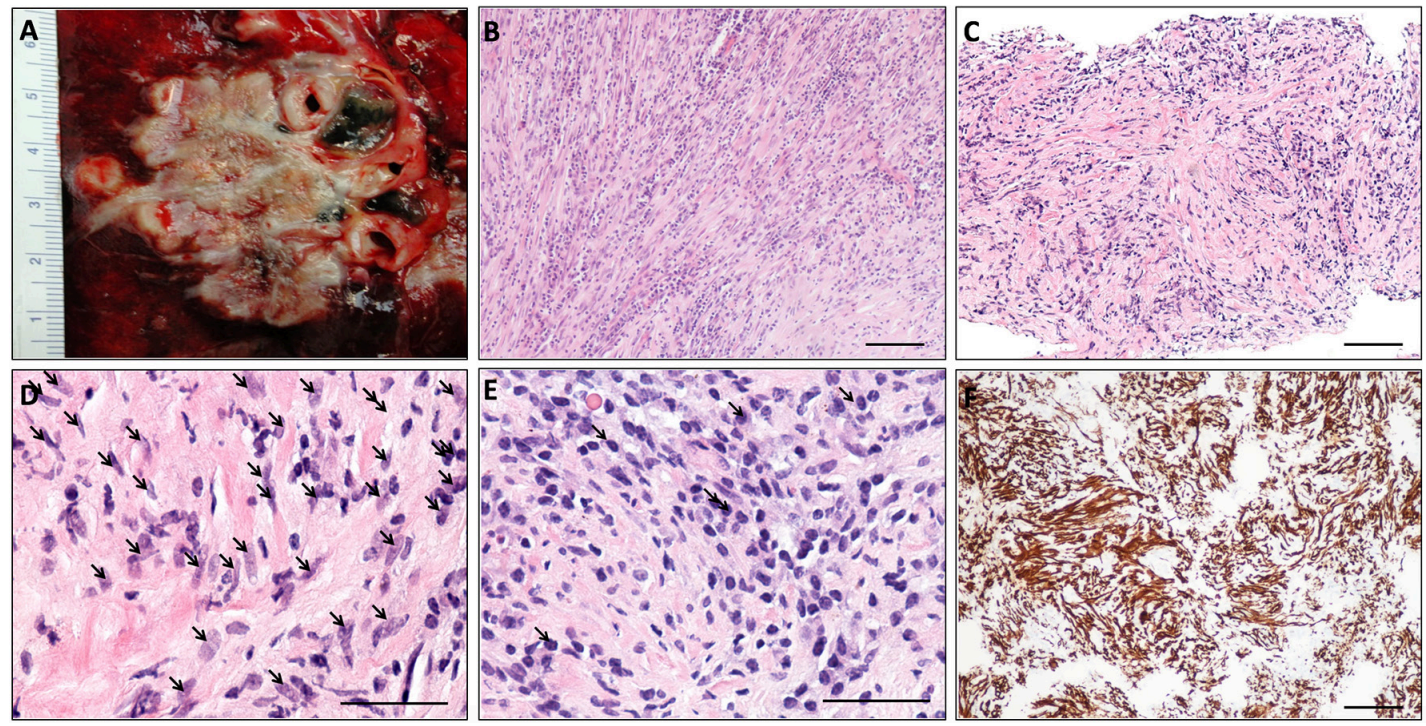

Figure 2 (A, B) Right upper lobe tumour resected in 2005. (A) Shows the cut surface and (B) the histology, consisting of a fibroinflammatory lesion. (C-F) Histology of the recurrent tumour in the left lower lobe. Sections show a fibroinflammatory lesion (C) similar to the original tumour (B), with areas of numerous myofibroblasts (D) and others with numerous plasma cells (E). (F) Antibodies to smooth muscle actin (brown stain), highlighting the myofibroblasts. Scale bars represent $100 \mu \mathrm{m}(B, C, F)$ and $50 \mu \mathrm{m}(D, E)$.

disease in a solitary lung presents a management dilemma due to limited surgical options. Prior case reports and case series have shown corticosteroids to stabilise disease and even induce remission. ${ }^{2}$ Due to the low recurrence rate after complete resection, it remains unknown if empiric medical therapy postsurgery could prevent long-term recurrence. This patient's disease recurrence responded well to steroid therapy, with symptomatic improvement and radiographic regression of the tumour. These changes persisted after 14 months of follow-up. This case supports the view that corticosteroid therapy may be effective in patients with unresectable disease and informs the need for longitudinal follow-up in patients with IMT due to the unpredictable risk of recurrence, despite complete resection and even pneumonectomy. Further study is required to determine optimal dose and duration of corticosteroid therapy, as well as identify potential alternatives to corticosteroids for long-term medical therapy.

Acknowledgements We thank the patient and his family for allowing us to document this unique clinical presentation.

Contributors DCV and KAJ conceived the project. DCV, MMK, AGL, GG and KAJ contributed important intellectual content. All authors contributed to and approved the final version of the manuscript. KAJ is the guarantor of this manuscript, taking full responsibility for its contents.

Funding The authors have not declared a specific grant for this research from any funding agency in the public, commercial or not-for-profit sectors.

Competing interests None declared.

Patient consent Obtained.

Provenance and peer review Not commissioned; externally peer reviewed.

Author note 1089

(C) Article author(s) (or their employer(s) unless otherwise stated in the text of the article) 2018. All rights reserved. No commercial use is permitted unless otherwise expressly granted.

\section{REFERENCES}

1 Kovach SJ, Fischer AC, Katzman PJ, et al. Inflammatory myofibroblastic tumors. J Surg Oncol 2006;94:385-91.

2 Schaeffer CJ, Minai OA, Sharma N, et al. Inflammatory myofibroblastic tumor of the lung: recurrence after steroid treatment. J Thorac Imaging 2008;23:191-3.

3 Goto T, Akanabe K, Maeshima A, et al. Surgery for recurrent inflammatory pseudotumor of the lung. World I Surg Oncol 2011;9:133. 